\title{
Implementación del software de Automatización de Bibliotecas y Centros de Documentación en la Unidad Académica de Ingeniería de Sistemas, Eléctrica y Electrónica
}

\section{Implementation of the software of Automation of Libraries and Documentation Centers in the Academic Unit of Systems Engineering, Electrical and Electronics}

\author{
Milton Alfredo Campoverde Molina*, Jenny Karina Vizñay Durán y Silvia Eulalia Vintimilla Jara \\ Unidad Académica de Tecnologías de la Información y la Comunicación (TIC) \\ Cuenca, Ecuador \\ *mcampoverde@ucacue.edu.ec
}

\begin{abstract}
Resumen
Esta investigación tiene como objetivo la implementación del software de Automatización de Bibliotecas y Centros de Documentación (ABCD) en la biblioteca de la Unidad Académica de Ingeniería de Sistemas, Eléctrica y Electrónica de la Universidad Católica de Cuenca, permitiendo unificar y gestionar la información bibliográfica en un solo repositorio digital de modo que los usuarios accedan a la misma a través del internet, para lo cual se crearon los módulos de: catalogación, administración, adquisición y préstamos, también las hojas de entrada para el ingreso de tesis, monografías y trabajos de investigación; luego de la implementación se aplica una encuesta a un grupo objetivo de 15 estudiantes y 5 profesores la misma que da como resultado que el 46,67\% de la colección de libros básicos de la carrera es buena, el 33,33\% es muy buena y los servicios de consultas online, préstamos, referencias electrónicas y búsquedas se cumple con el sistema en un $84 \%$ y un $16 \%$ no se cumple de acuerdo a los datos de los encuestados.
\end{abstract}

Palabras clave: ABCD, Bibliotecas, Centros de Documentación, Software.

\begin{abstract}
The aim of this research is to implement the Automation Software for Libraries and Documentation Centers $(A B C D)$ in the library of the Academic Unit of Systems, Electrical and Electronics Engineering of Catholic University of Cuenca, allowing to unify and manage the bibliographic information in a single digital repository so that users can access it through the internet. For this purpose the following modules were created: cataloging, administration, acquisition and loans, as well as the entry sheets for submitting thesis, monographs and research work; After the implementation, a survey is applied to a target group of 15 students and 5 teachers which shows as result that $46.67 \%$ of the basic books' collection of the career is good, $33.33 \%$ is very good and that the online consultation services, loans, electronic references and searches are met by the system in $84 \%$ while $16 \%$ is not met according to the respondents' data.
\end{abstract}

Key words: ABCD, Libraries, Documentation Centers, Software.

\section{INTRODUCCIÓN}

$\mathrm{L}$ A misión de los centros bibliotecarios ha sido coleccionar y organizar materiales para facilitar el uso por parte de sus usuarios. Cuando la información estaba disponible únicamente en formato papel y se necesitaban grandes áreas físicas para almacenarla, contar con profesionales experimentados en la clasificación y organización de las colecciones era una necesidad obvia [1]. El auge de la Sociedad de la Información, ha dado lugar a la incorporación progresiva de los profesionales de la información y la documentación en los nuevos ámbitos de tratamiento, gestión y difusión de información digital. Con ellos, han surgido herramientas informáticas de desarrollo libre y gratuito que gestionan de manera eficaz los contenidos digitales [2].

En su cumplimiento, las bibliotecas universitarias han transitado desde su forma más tradicional hasta mostrar grandes colecciones en versión digital [3]. Todos los logros actuales en materia de automatización no son sino fruto de un proceso largo y costoso que comenzó allá por los años 60, encabezado por la LC de EEUU, cuyo informe de 1963 sentó las bases para el nacimiento del formato MARC. En los años 70, con la aparición de los microprocesadores, la 
automatización se dirigió fundamentalmente a los catálogos, lo que fomentó el desarrollo de iniciativas cooperativas y denotó la necesidad de unificar criterios normativos. En los años 80, aparecieron los OPAC y los primeros Sistemas Integrados, como Dobis/Libis, Aleph o SABINI. Desde los años 90 en adelante, la madurez ha ido llegando en forma de avances tecnológicos, estándares e innovaciones varias, observadas, por ejemplo, en los Sistemas de Gestión de Bases de Datos (SGBD), relacionales o documentales, en los grandes Sistemas Integrados de Gestión Bibliotecaria, que han extendido la automatización a tareas bibliotecarias clave, y, sobre todo, en Internet y la WWW, cuya evolución ha permitido progresos y posibilidades antes impensables [4].

La situación de las bibliotecas del futuro vendrá determinada por varios factores, los cambios de paradigma en las arquitecturas tecnológicas y del entorno jurídico y empresarial en materia de propiedad intelectual y derechos de autor. El progreso de las bibliotecas también dependerá de la situación económica y de su acceso a los recursos necesarios, incluyendo las infraestructuras de red e informáticas, disponibilidad de internet de banda ancha, así como del grado de interés y capacidad de los usuarios para aprovechar los servicios informáticos y de la Web, ...y muchos otros factores [5].

En la actualidad las bibliotecas, especialmente aquellas que trabajan en áreas especializadas - universitarias y de investigación - tienen un reto importante ante sí para poder desarrollar sus potencialidades como verdaderos centros de apoyo a la investigación, que proporcionan servicios documentales a la medida de las necesidades de sus usuarios [6]. Sabiendo que las funciones prioritarias de la biblioteca universitaria son: servir de apoyo a la formación, la investigación y la docencia [7]. Lo que motiva, implementar un sistema de automatización de bibliotecas que posea un conjunto de módulos que abarcan las actividades bibliotecarias más importantes, los cuales están relacionadas entre sí, ya que comparten una misma base de datos, aunque dichos módulos tienen funciones distintas están unificados para facilitar su control, y de ésta manera ayudar a mejorar la eficiencia y eficacia de los procesos, servicios y de la gestión general de la biblioteca [8].

Los servicios deben estar enfocados en los planes y programas académicos de cada institución, promover y facilitar el uso de la información, sin importar su soporte, y procurar que llegue a todos sus usuarios, sin olvidar que, además, deben apoyar la misión, la visión y las metas de la institución. Estos servicios incluyen: asesoramiento, asistencia personalizada y consulta o referencia, horario, atención a usuarios y otros [9].

En las universidades británicas de Bath, Bradford, East London, Glamorgan, Hertfordshire, Leeds Metropolitan, London Guidhall, Luton y Sheffield Hallam, han fundado en los últimos años centros de recursos para el aprendizaje que responden a una estructura que integra servicios y recursos bibliotecarios, tecnológicos y audiovisuales; sis- temas de información; e instalaciones y medios para la edición electrónica y la creación de materiales interactivos, a fin de dar el debido soporte a las necesidades docentes y de aprendizaje de la comunidad universitaria [10].

La Ley Orgánica de Educación Superior del Ecuador (LOES) [11], Título VII, Capítulo II, Sección Tercera, en el Art. 143 manifiesta que, las instituciones de educación superior públicas y particulares desarrollarán e integrarán sistemas interconectados de bibliotecas a fin de promover el acceso igualitario a los acervos existentes, y facilitar préstamos e intercambios bibliográficos. Participarán en bibliotecas digitales y sistemas de archivo en línea de publicaciones académicas a nivel mundial. Asimismo en el Art. 144 que, todas las instituciones de educación superior estarán obligadas a entregar las tesis que se elaboren para la obtención de títulos académicos de grado y posgrado en formato digital para ser integradas al Sistema Nacional de Información de la Educación Superior del Ecuador para su difusión pública respetando los derechos de autor.

La Unidad Académica de Ingeniería de Sistemas, Eléctrica y Electrónica de la Universidad Católica de Cuenca oferta las carreras de Ingeniería de Sistemas e Ingeniería Eléctrica, la misma que dispone de una biblioteca común en cuanto al espacio físico, sin embargo el material bibliográfico se administra en forma independiente. La problemática detectada en la biblioteca se debe a que los procesos inherentes a la misma se realizan en dos ambientes diferentes; por un lado el software PMB (PhpMyBibli) permite realizar el ingreso de nuevos libros a inventario, préstamos, devoluciones, reservas y reportes; en cambio las tesis, monografías y trabajos de investigación se encuentran en un repositorio DSpace; evidenciando claramente que la información de la biblioteca no se encuentra centralizada en un mismo repositorio digital.

El objetivo de esta investigación es implementar el software de Automatización de Bibliotecas y Centros de Documentación (ABCD), en la Unidad Académica de Ingeniería de Sistemas, Eléctrica y Electrónica de la Universidad Católica de Cuenca, que integre la información bibliográfica en un solo repositorio digital.

\section{MARCO TEÓRICO}

$\mathrm{ABCD}$ es un software de gestión integrado de bibliotecas basado en la web que comprende las principales funciones básicas de la biblioteca. Este tipo de aplicación de biblioteca es una aspiración largamente sostenida de la comunidad ISIS, ya que la primera versión de MS-DOS salió hace más de 20 años. Varios sistemas de automatización de bibliotecas se han desarrollado durante este período y se encuentran todavía en funcionamiento en todo el mundo. BIRME EMP anterior sistema estaba limitado a los servicios de circulación. Las principales características de ABCD son la cobertura de las principales funciones de la biblioteca, su centralidad web y su desarrollo y mantenimiento, mediante la metodología de Free y Open Source Software [12]. 
Este sistema se distribuye con bases de datos MARC 21, CEPAL o LILACS, permitiendo el uso de cualquier formato de catalogación permitiendo migrar bases de datos desde WinIsis y/o Excel. El ABCD apunta a proporcionar a la comunidad de usuarios de la familia ISIS, un Sistema integrado para bibliotecas, incluso este software permite las exigencias de las bibliotecas universitarias, otras de las bondades del software es que corre en diferentes navegadores: IE, FF, Ópera, Chrome, Safari, etc. Y en cualquier PC con Windows o UNIX/Linux [13].

BIREME, expresa que ABCD tiene la capacidad de diseñar y adaptarse a cualquier estructura, sin restringir funcionalidades avanzadas, abriendo las puertas al manejo integrado de catálogos de bibliotecas, archivos y museos, así como a bibliotecas virtuales; en realidad este software es aplicable a cualquier tipo de bibliotecas y centros de documentación, que trabajen o no con ISIS, ya que integran todas las aplicaciones necesarias para la automatización online de su trabajo, sin tener que modificar sus bases actuales, sólo se tendría que migrarlas a ABCD [13].

El módulo "central" de ABCD [12] comprende módulos para administración de bases de datos (creación de bases de datos, edición de estructuras de bases de datos, utilitarios para bases de datos) catalogación, adquisición, circulación/préstamos y estadísticas. Se está preparando un módulo de gestión de tesauros como parte del módulo de catalogación para una base de datos específica con estructura de tesauro, y con control de consistencia de los niveles jerárquicos. Como parte de este "módulo central" también deseamos mencionar los servicios de importación y exportación, impresión, y herramientas de bases de datos, como bloquear/desbloquear y cambios globales a los campos de los registros; el módulo de préstamos se denomina "básico" ya que está completamente integrado con los otros módulos centrales de ABCD, utilizando la misma tecnología: bases de datos ISIS, ISIS Script y PHP. En cuanto a su funcionalidad sin embargo, difícilmente se podría llamar "básico": este módulo toma como punto de partida los objetos creados por el módulo de adquisiciones en la base de datos "copia", para aplicar las normas en todo tipo de "operaciones" sobre ellos: la entrega a un usuario, la devolución, la reserva, renovación de préstamos. Se pueden definir normas para todo tipo de operaciones y se aplicarán de acuerdo a la categoría de objeto en combinación con la categoría de usuario. Las categorías para los objetos y para los usuarios se pueden definir "ad libitum" con un número especificado de objetos, horas / días (teniendo en cuenta un calendario específico para la biblioteca), multas y condiciones de renovación para cada combinación objeto/usuario. El menú principal de este módulo de préstamos tiene tres secciones:

1) Transacciones: aquí se tratan las transacciones reales de préstamos (préstamo de un libro a un usuario, la devolución, reservas).

2) Bases de datos: aquí se pueden acceder y gestionar las bases de datos en la que el sistema de préstamos se basa: los prestatarios o los usuarios, las transacciones, las reservas y las multas.

3) Configuración del sistema de préstamos: aquí se pueden definir las "reglas" para las combinaciones de tipos de objetos con las categorías de usuarios y calendarios, moneda, etc.

Asimismo, el módulo de adquisiciones [12] trata de la administración de objetos recientemente adquiridos, y funciones de pre-catalogación. Los objetos pre-catalogados pueden ser almacenados como objetos para el módulo de préstamos, después de adquiridos. De esto se trata la "integración” en ABCD. El módulo de adquisición tiene las siguientes funciones lógicas:

1) Sugerencias: el proceso inicial de obtener objetos.

2) Órdenes de compra: la adquisición real de objetos.

3) Bases de datos: gestión de las 4 bases de datos relacionadas con la adquisición (sugerencias, proveedores, pedidos y copias).

4) Administración: configuración, estadísticas e informes, expurgo (objetos descartados).

\section{Materiales y/O Métodos}

\section{A. Análisis Situacional}

Esta investigación inicia con la formulación de una encuesta para determinar las necesidades y servicios que ofrece la biblioteca en función de 5 criterios:

1) Datos personales.

2) Catalogación de los libros en la biblioteca.

3) Infraestructura.

4) Servicios de la biblioteca.

5) Atención en la biblioteca.

Luego se realizó la aplicación de la encuesta a 50 docentes y 249 estudiantes de las carreras de Ingeniería de Sistemas e Ingeniería Eléctrica de la Universidad Católica de Cuenca, en base a los criterios evaluados se presenta los resultados obtenidos de la tabulación de los datos de la encuesta de los criterios 2,3 y 4 , que se toma como referencia para esta investigación:

\section{Catalogación: ¿Cómo es la colección de libros básicos en la carrera, existente en biblioteca?}

Los resultados se pueden observar en la Tabla I y II:

TABLA I

COLECCIÓN DE LIBROS BÁSICOS EN LA CARRERA (DOCENTES).

\begin{tabular}{|c|c|c|}
\hline & Frecuencia & Porcentaje \\
\hline Muy Mala & 19 & $38,0 \%$ \\
\hline Mala & 8 & $16,0 \%$ \\
\hline Regular & 15 & $30,0 \%$ \\
\hline Buena & 8 & $16,0 \%$ \\
\hline Muy Buena & 0,0 & $0,0 \%$ \\
\hline Total & 50 & $100,0 \%$ \\
\hline
\end{tabular}


TABLA II

Colección de Libros básicos EN La CARRERA (ESTUdiantes).

\begin{tabular}{|c|c|c|}
\hline & Frecuencia & Porcentaje \\
\hline Muy Mala & 13 & $5,2 \%$ \\
\hline Mala & 58 & $23,3 \%$ \\
\hline Regular & 108 & $43,4 \%$ \\
\hline Buena & 55 & $22,1 \%$ \\
\hline Muy Buena & 15 & $6,0 \%$ \\
\hline Total & 249 & $100,0 \%$ \\
\hline
\end{tabular}

Según los resultados obtenidos de los docentes se determina que un $38,0 \%$ de la colección de libros básicos en la carrera es muy mala y el $30,0 \%$ es regular en cambio los estudiantes establecen que el $43,4 \%$ es regular y el $23,3 \%$ es mala; tomando como referencia sólo los dos porcentajes más altos de los resultados en ambos casos.

Realizando una correlación de los datos de la encuesta aplicada a estudiantes y docentes se determina que el $41,14 \%$ de la colección de libros básicos en la carrera es Regular, el 22,07\% es Mala, el 21,07\% es Buena, el $10,70 \%$ es Muy Mala y el 5,02\% es Muy Buena.

2. Catalogación: ¿Cómo es la colección de libros básicos en la Biblioteca?

Los resultados se pueden observar en la Tabla III y IV:

TABLA III

COLECCIÓN DE LIBROS BÁSICOS EN LA BIBLIOTECA (DOCENTES)

\begin{tabular}{|c|c|c|}
\hline & Frec. & Porcent. \\
\hline Colección Obsoleta (Antigua) & 25 & $50,0 \%$ \\
\hline Número de copias insuficientes & 20 & $40,0 \%$ \\
\hline Títulos inexistentes & 5 & $10,0 \%$ \\
\hline Total: & 50 & $100,0 \%$ \\
\hline
\end{tabular}

TABLA IV

COLECCIÓN DE LIBROS BÁSICOS EN LA BIBLIOTECA (ESTUDIANTES).

\begin{tabular}{|c|c|c|}
\hline & Frec. & Porcent. \\
\hline Colección Obsoleta (Antigua) & 90 & $29,8 \%$ \\
\hline Número de copias insuficientes & 146 & $48,3 \%$ \\
\hline Títulos inexistentes & 66 & $21,9 \%$ \\
\hline Total: & 302 & $100,0 \%$ \\
\hline
\end{tabular}

Con respecto a cómo es la colección de libros básicos en la biblioteca, los docentes determinan que un 50,0\% de la colección es Obsoleta (Antigua) y el 40,0\% que el número de copias son insuficientes en cambio los estudiantes establecen que el $48,3 \%$ el número de copias son insuficientes y el $28,9 \%$ que la colección es Obsoleta (Antigua); asimismo, tomando como referencia sólo los dos porcentajes más altos de los resultados en ambos casos.

Realizando una correlación de los datos de la encuesta aplicada a estudiantes y docentes se determina que el
29,80\% de la colección es Obsoleta (Antigua), el 48,34\% que el número de copias son insuficientes y el $21,85 \%$ que hay títulos inexistentes en biblioteca.

\section{Infraestructura: El equipamiento tecnológico en la Bi- blioteca es:}

Los resultados obtenidos se puede observar en las Figuras 1 y 2 :

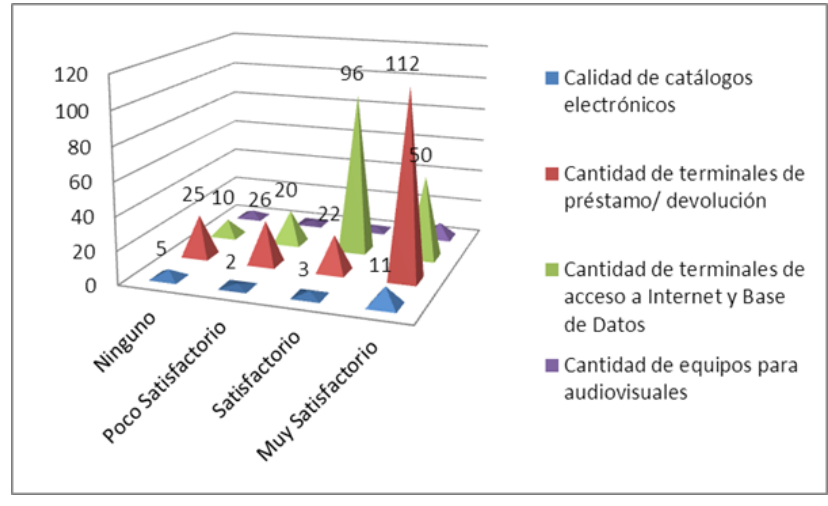

FIG. 1. El equipamiento tecnológico en la Biblioteca (docentes).

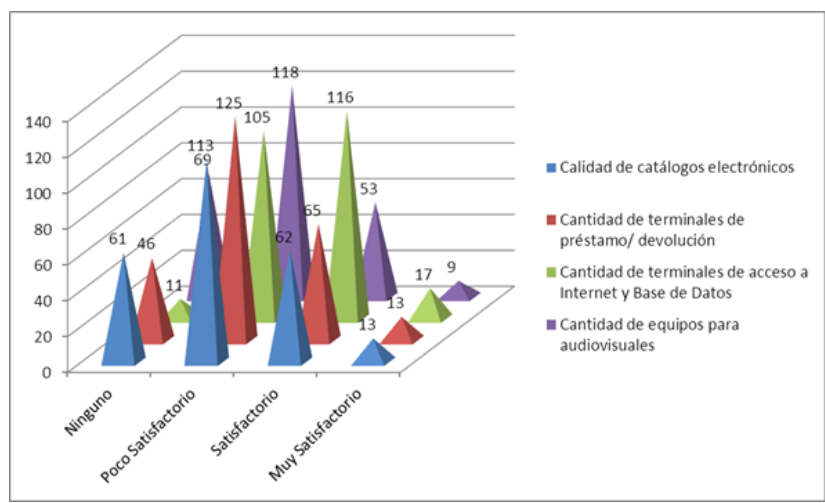

FIG. 2. El equipamiento tecnológico en la Biblioteca (estudiantes).

En cuanto al equipamiento tecnológico en la Biblioteca, los docentes determinan que es Muy Satisfactorio y en cambio los estudiantes establecen que es Poco Satisfactorio.

\section{Servicios: ¿Cuáles de los siguientes servicios existen en la biblioteca?}

Los resultados obtenidos se puede observar en las Figuras 3 y 4 :

Los estudiantes determinan que el $49 \%$ de estos servicios NO se cumplen en la biblioteca como se ve en la Figura 3 y el $51 \%$ que estos servicios SI se cumplen, en cambio los docentes determinan que el $41 \%$ de estos servicios NO se cumplen en la biblioteca como se ve en la Figura 4 y el $59 \%$ que estos servicios SI se cumplen.

Realizando una correlación de los datos de la encuesta aplicada a estudiantes y docentes se determina que el $45 \%$ 


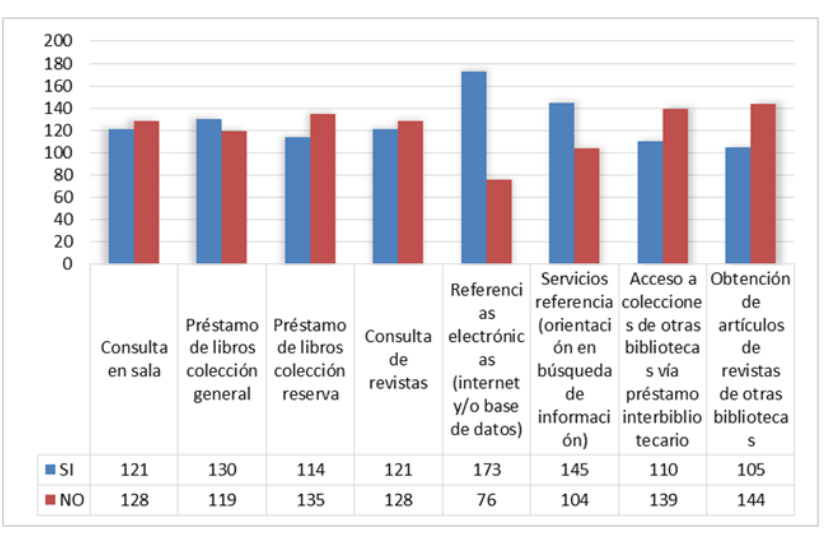

FIG. 3. Servicios que ofrece la Biblioteca (estudiantes).

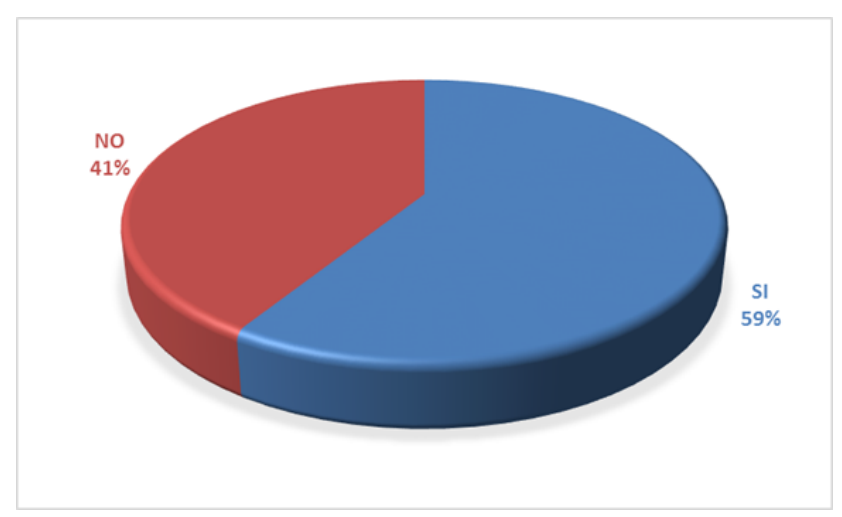

FIG. 4. Servicios que ofrece la Biblioteca (docentes).

de estos servicios NO se cumplen en la biblioteca y el $55 \%$ SI se cumplen en la biblioteca.

\section{B. Instalación y configuración del Software $A B C D$}

Para la implementación de este software se realizó la instalación de un Servidor con Windows 2008 Server, el mismo que cuenta con las características recomendadas de hardware:

1) Procesador de $2 \mathrm{GHZ}$ por lo menos.

2) Memoria RAM por lo menos de $2 \mathrm{~GB}$.

3) Espacio en disco duro 100 Gb o más.

4) Monitor VGA con resolución de 800 x 6000 o superior.

Posteriormente se instalaron los requerimientos de software para la configuración del ABCD.

Windows o Linux [14]:

1) Apache 2.2.x.

2) PHP 5.2.X.

3) YAZ.

4) Mysql 5.1 o superior (solamente para EmpWeb).

5) Java 1.5 (solamente para EmpWeb).

La descarga del software se puede realizar de la siguiente URL http://wiki.bireme.org/es/index.php/ABCD_ Recursos/
Para la instalación hay disponibles dos tipos de paquetes: Instalación asistida (Wizard), o no asistida (.zip) en esta instalación se utilizó la no asistida.

Luego de haber descargado el Software ABCD se descomprime la carpeta y pega en la unidad $\mathrm{C}$ del computador, posteriormente se ingresa dentro de la carpeta llamada ABCD y da doble clic en $A B C D \_s t a r t$ para que se visualice la página de inicio del $\mathrm{ABCD}$ en el navegador como se observa en la Figura 5.

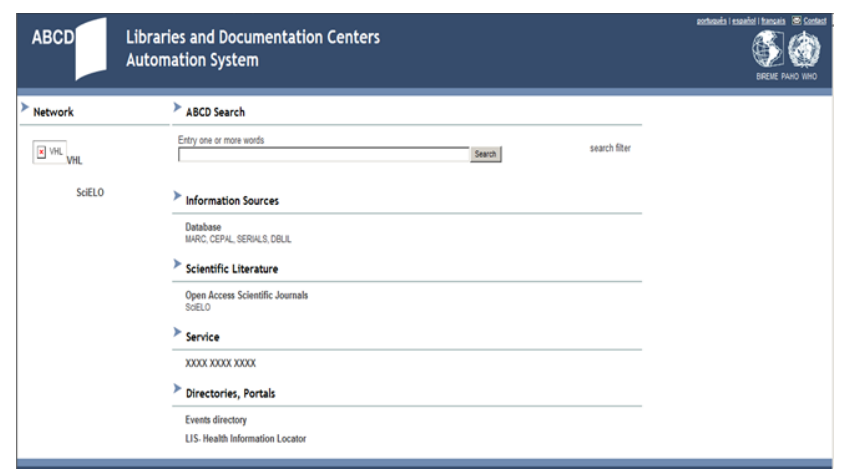

FIG. 5. Página de inicio de ABCD.

Para ingresar al ABCD en el navegador se coloca http://localhost:9090/, y luego en el texto de usuario se digita $a b c d$ y en la contraseña $a d m$ como se visualiza en la Figura 6.

\section{Identificación}

\section{abcd}

\section{Clave \\ 10}

Idioma

español

Abrir en una nueva ventana

Entrar

FIG. 6. Inicio de Sesión ABCD.

EL ABCD consta de varios módulos que conforman las diferentes tareas administrativas del bibliotecario, estos módulos son relativamente independientes [15].

- Gestión de bases de datos, creación y modificación de la estructura.

- Administración de usuarios. 
- Generación de formularios de búsquedas.

- Estadísticas.

- Servidores de catalogación Z39.50.

- Utilitarios.

- Configuraciones.

- Gestión de adquisiciones.

- Gestión de préstamos/circulación.

\section{Implementación del Módulo de Catalogación}

\section{Hojas de Entrada}

ABCD permite la creación de diferentes hojas de entrada dependiendo de las necesidades bibliográficas de los centros de documentación en el caso de la biblioteca de las carreras de Ingeniería de Sistemas e Ingeniería Eléctrica se diseñaron las hojas de entrada: Monográfica, Analítica de Monográfica, Colección, Monográfica de Colección, Publicación Seriada, Analítica de Seriada y Tesis con sus respectivos campos y secuencia de los mismos para el llenado de los registros.

La creación de las hojas de entrada se realizó seleccionando la base de datos Formato CEPAL y a continuación clic en Actualizar definición de base de datos como se observa en la Figura 7.

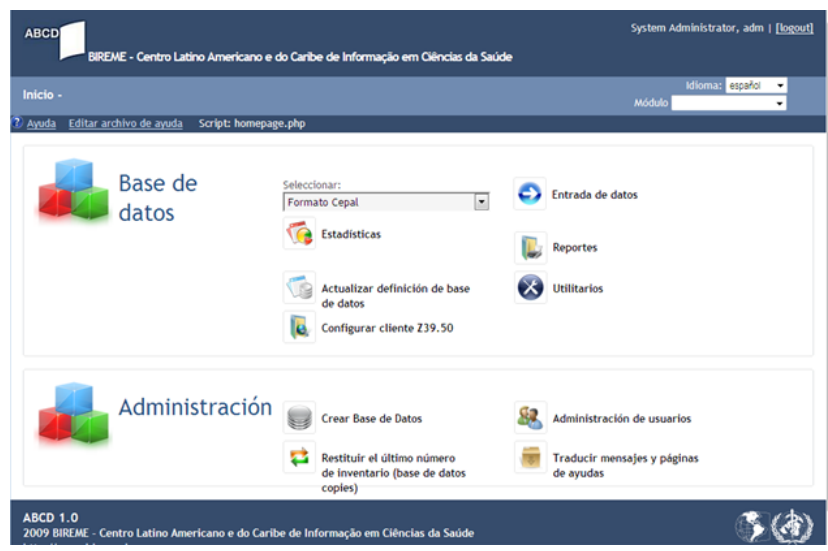

FIG. 7. Creación de las Hojas de Entrada.

En la siguiente ventana se da clic sobre hoja de entrada y a continuación se selecciona la hoja de entrada a ser creada, la misma que puede ser de cualquiera de los siguientes tipos:

1) Monográfica.

2) Analítica de monográfica.

3) Colección.

4) Monográfica de colección.

5) Publicación seriada.

6) Analítica de seriada.

7) Tesis.

8) Tabla de contenido.

En esta misma ventana se encuentran todos los campos declarados en nuestra FDT (base de datos CEPAL), dependiendo de la hoja de entrada se debe seleccionar los campos del cuadro izquierdo y agregar al cuadro derecho, cuando ya se hayan definido todos los campos se debe dar un nombre y descripción a la hoja de entrada y guardar como se observa en la Figura 8.

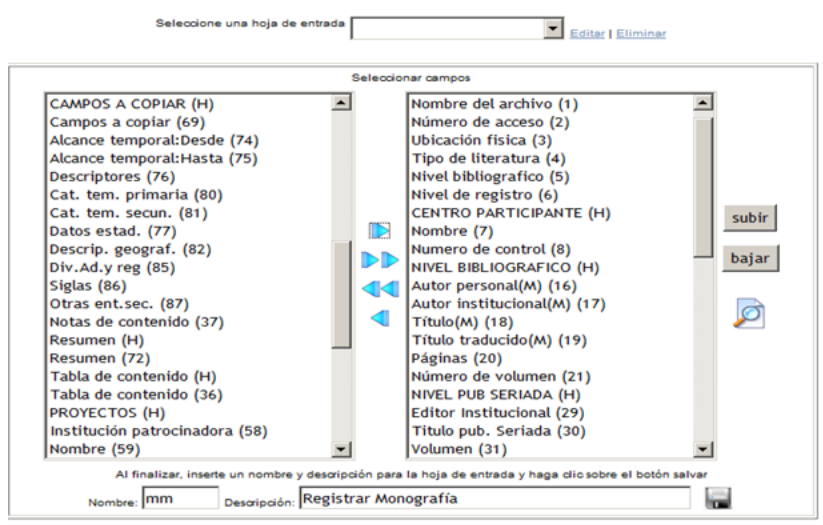

FIG. 8. Definición de los campos en las Hojas de Entrada.

Luego se ingresa dentro de tipo de registro y coloca el nombre de la hoja de entrada con su respectiva etiqueta y tipo de registro, y posteriormente se da clic sobre el botón actualizar y con esto se visualiza las hojas de entrada como se ve en la Figura 9:

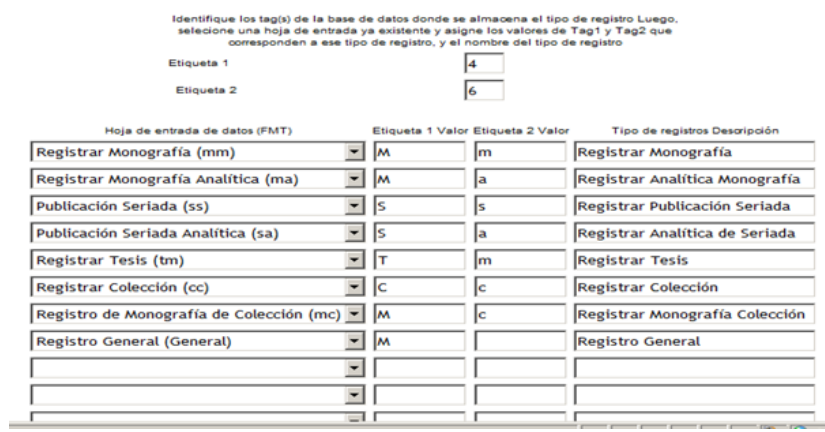

FIG. 9. Hojas de Entrada Creadas.

\section{Entrada de datos}

Esta opción permite el ingreso de nuevos registros, su edición o eliminación de los mismos a través de cada una de las hojas de entrada a la base de datos, para su ingreso se selecciona Formato CEPAL y a continuación se da clic en Entrada de Datos.

En esta etapa se realizó el ingreso de la información de las tesis, monografías y trabajos de investigación a través de sus hojas de entrada al sistema.

\section{Utilitarios}

En esta opción ABCD ofrece algunas operaciones de administración de la bases de datos, en este proyecto se utilizaron las siguientes [12]: 


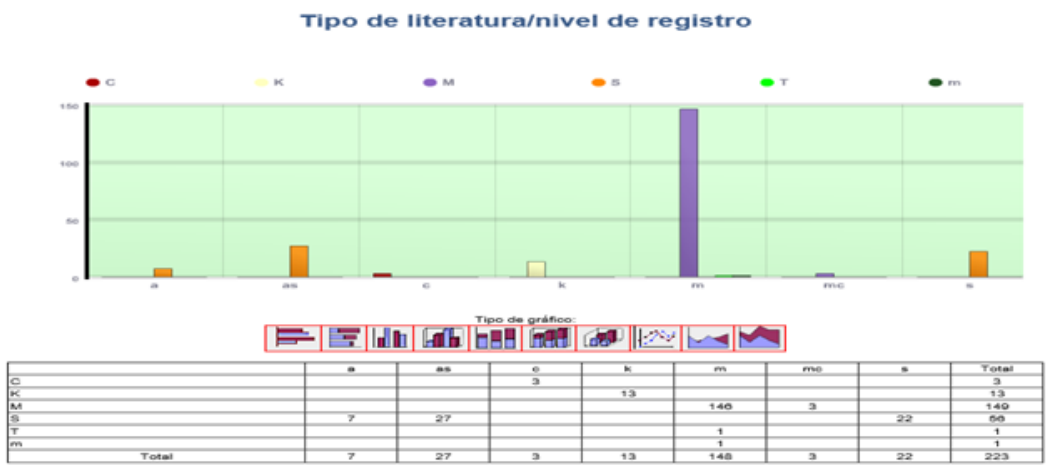

FIG. 10. Estadística Animada.

- Inicializar base de datos: significa eliminar todos los registros en la base de datos pero sin modificar su estructura.

- Eliminar base de datos: significa borrar totalmente la base de datos con todos los archivos y las carpetas correspondientes en la carpeta de ABCD /bases.

- Bloquear base de datos: significa evitar que otros usuarios hagan cambios en los registros (ingreso de datos).

- Desbloquear base de datos: desde luego significa ponerla a disposición nuevamente para otros usuarios.

Para ingresar a esta opción se selecciona Formato $C E$ $P A L$ y a continuación se da clic en Utilitarios.

La administración de la base de datos durante todo el proceso estuvo a cargo del usuario administrador.

\section{Estadísticas}

En esta opción al igual que las anteriores se ingresa seleccionando Formato CEPAL y a continuación al dar clic sobre Estadísticas se puede realizar las siguientes funciones:

- Usar una tabla existente.

- Crear una tabla.

- Generar salida.

- Configuración de las estadísticas.

Se crearon tablas que generen estadísticas de los préstamos, devoluciones, ingreso de los estudiantes al sistema, adquisiciones, órdenes de compra y en otros casos se utilizaron tablas existentes para sacar estadísticas de cada una de las mismas como se observa en la Figura 10:

\section{Reportes}

Se pueden generar reportes de rangos, por expresión de búsqueda, clave de ordenamiento utilizando las siguientes opciones:

- Usar un formato existente.

- Crear un formato (seleccione los campos que desea reportar).

- Generar salida.

- Guardar formato.
El formato de salida de los reportes incluye: procesador de texto, procesador de hojas de cálculo, vista previa y txt.

Además se pueden guardar los formatos de salida cuando se requiere sacar el mismo reporte de forma constante.

\section{Búsquedas}

Formulario de búsqueda avanzada: Es útil en el módulo de catalogación, tiene el propósito de que el bibliotecario realice una búsqueda eficiente y rápida de un registro.

Cabe mencionar que las búsquedas se pueden configurar de acuerdo a las necesidades del bibliotecario.

\section{Implementación del Módulo de Administración \\ 1. Crear Base de Datos}

La creación de una Base de Datos en ABCD se puede observar en la Figura 11:

\begin{tabular}{ll}
\hline (3) Ayuda Editar ayuda & Script: menu_creardb.php \\
$\begin{array}{l}\text { Nombre de la base de } \\
\text { datos }\end{array}$ & repositoriobibliotecasis_db \\
Descripción & Base de datos de la Biblioteca \\
Crear desde: & Formato Cepal
\end{tabular}

FIG. 11. Creación de una Base de Datos en ABCD.

El software ABCD ya tiene creadas las bases de datos para proveedores, adquisiciones, préstamos y órdenes de compra.

\section{Administrar Usuarios}

Esta ventana permite administrar los usuarios del sistema y crear/editar perfiles.

Los perfiles de usuario con los cuales cuenta ABCD son los siguientes:

1) Administrador del Sistema: el único perfil que permite crear nuevas bases de datos y administrar las cuentas de usuarios. 
2) Administrador de Base de Datos: este perfil permite hacer cambios en la estructura de las bases de datos y registros.

3) Operador de Base de Datos: este perfil permite editar los registros de la base de datos (catalogador).

4) Administrador de adquisiciones: este perfil permite usar las funciones de adquisiciones de ABCD, no se pueden hacer cambios a las bases de datos.

5) Operador de adquisiciones: este perfil permite editar los registros de adquisiciones en la base de datos (catalogador).

6) Administrador de préstamos: este perfil permite usar las funciones de préstamo de $\mathrm{ABCD}$, no se pueden hacer cambios a las bases de datos.

7) Operador de préstamos: este perfil permite editar los registros de préstamos en la base de datos (catalogador).

También a cada uno de estos perfiles se puede personalizar su funcionamiento quitando o agregando funciones de acuerdo a las necesidades o requerimientos de seguridad, además se pueden crear nuevos perfiles.

En la Figura 12 se puede observar los campos a rellenar para crear un usuario:

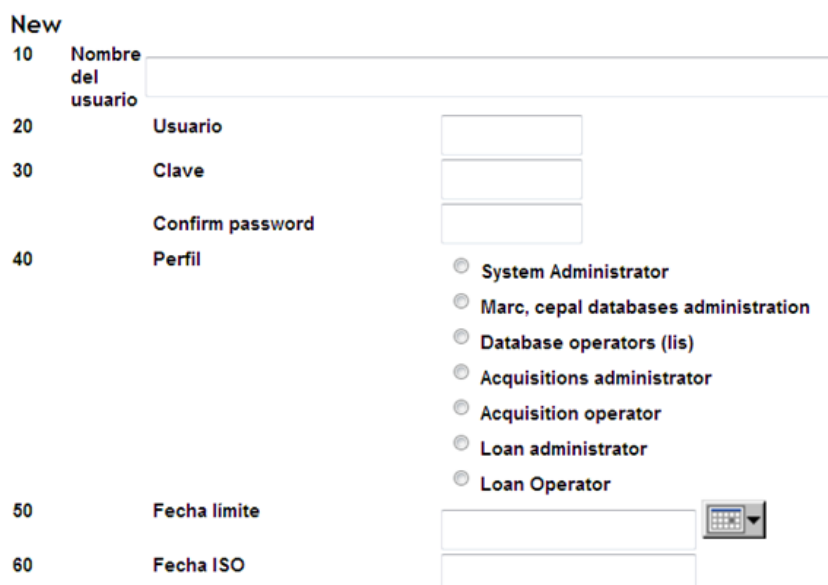

FIG. 12. Formulario para la creación de un Usuario.

Los usuarios con los que se trabajó en la implementación del software ABCD se puede observar en la Tabla V:

TABLA V

USUARIOS UTILIZADOS PARA LA CREACIÓN DE LAS BASES DE DATOS E INGRESO DE REGISTROS.

\begin{tabular}{|c|c|}
\hline Perfil & Identificador \\
\hline Administrador del Sistema & andres \\
\hline Operador de Base de Datos & alfredo \\
\hline Catalogador & henry \\
\hline Catalogador & joffre \\
\hline Catalogador & ivan \\
\hline Catalogador & alex \\
\hline
\end{tabular}

\section{E. Implementación del Módulo de Adquisiciones}

Se selecciona la base de datos que es necesaria para el módulo de este caso: Adquisitions, Copies como se puede ver en la Figura 13:

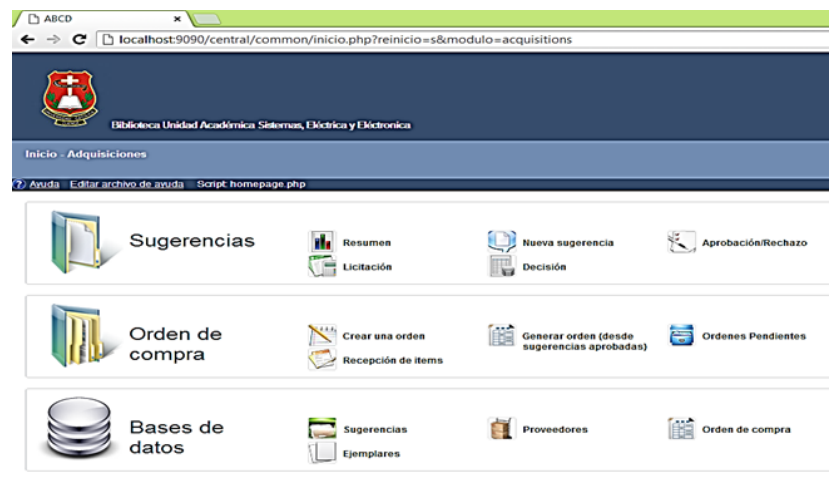

FIG. 13. Selección de la base de datos para la creación del módulo Adquisiciones

Permite tener el resumen de libros que están en proceso de adquisición, pendientes, aprobados, rechazados, en licitación, proveedores elegidos, orden de compra de ítems recibidos y procesos de compras.

En este módulo se realizó el ingreso del inventario de libros de la biblioteca al sistema con la ayuda de los estudiantes investigadores.

\section{F. Implementación del Módulo de Préstamos}

El módulo de préstamos ofrece un manejo avanzado con algunas características extra para organizaciones más grandes y complejas. Provee además un módulo dirigido a los usuarios, basada en tecnología de servicios Web, que puede ser usado para reemplazar los módulos integrados de préstamo en caso de contar con muchas dependencias y altos volúmenes de transacciones [16].

La opción de transacciones permite prestar, reservar, devolver, renovar, suspender/multas, estado de un ítem, estado de cuenta, histórico del usuario y reportes de los libros como se puede observar en la Figura 14.

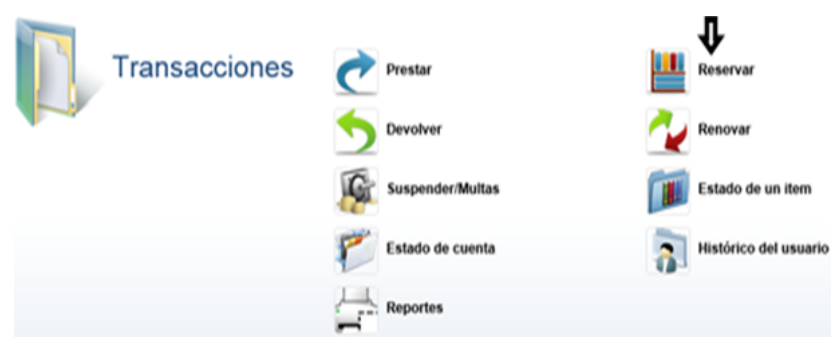

FIG. 14. Transacciones de prestamos y devoluciones.

Para prestar un libro se introduce o selecciona el código del libro desde inventario, el código de usuario desde usuarios y a continuación se da clic en prestar. 
De igual forma el usuario puede realizar la reserva de un determinado libro a través del Internet.

Para la devolución se ingresa el código del libro y da clic en devolver y automáticamente se guarda la información de la fecha de la devolución así como el tiempo que estuvo prestado el mismo.

La renovación ayuda a extender el plazo para la devolución del libro.

Si un libro no es devuelto a tiempo, se tiene la opción de suspender o multar al usuario que ha incumplido con el plazo para su devolución, para ello se ingresa al menú suspender/multas, se digita el ID del usuario y se pone buscar, luego se llena la ficha con el tipo de sanción, fecha, días de suspensión o valor a pagar, motivo, comentarios y por ultimo se da clic en actualizar para guardar la información en la base de datos.

Estado de cuenta, permite verificar la situación de determinado usuario, es decir, cuántos libros ha pedido, cuánto tardó en devolverlos, etc. se puede verificar su historial introduciendo el código de usuario o a su vez el código de un libro.

Si se necesita saber si un libro está prestado, cuándo lo devolvieron o simplemente saber algunas de sus características se da clic en estado de un ítem después de haber ingresado o escogiendo el código del libro desde la lista.

Los registros de un usuario como la cantidad de veces que pidió un libro, el tiempo, multas y más se pueden ver en Histórico del usuario, ingresando desde el menú principal.

Además se pueden emitir reportes de cada una de las transacciones antes mencionadas.

Esto ha mejorado notablemente la gestión de la biblioteca, además se dispone de reportes actualizados de los libros prestados, reservados, devueltos, pendientes, etc. y su disponibilidad en la web es 24/7.

\section{RESUltados Y Discusión}

En la Catalogación: ¿Cómo es la colección de libros básicos en la carrera, existente en biblioteca? en el análisis situacional de la encuesta aplicada a estudiantes y docentes se determina que el $41,14 \%$ de la colección de libros básicos en la carrera es Regular, el $22,07 \%$ es Mala, el $21,07 \%$ es Buena, el 10,70\% es Muy Mala y el 5,02\% es Muy Buena. Mientras que, luego de haber realizado la implementación del software ABCD y aplicado la encuesta a un grupo objetivo de 15 estudiantes y 5 profesores de las carreras, se obtiene como resultado que el 46,67\% de la colección de libros básicos en la carrera es Buena, el $33,33 \%$ es Muy Buena, el 20,00\% es Regular, el 0,00\% es Mala y el 0,00\% es Muy Mala. Existiendo un incremento significativo en los resultados.

En cuanto al equipamiento tecnológico en la Biblioteca los docentes determinan que es Muy Satisfactorio y los estudiantes que es Poco Satisfactorio en el análisis situacional, mientras que luego de haber realizado la implementación del software ABCD y aplicado la encuesta a un grupo objetivo de 15 estudiantes y 5 profesores de las carreras, se obtiene como resultado de los docentes Muy Satisfactorio y de los estudiantes Satisfactorio. Este resultado se justifica por la cantidad de catálogos electrónicos y terminales de préstamo/devolución en la biblioteca a través del internet.

Asimismo, se realizó un análisis comparativo de los servicios que ofrece la biblioteca, agrupado los indicadores de la siguiente manera:

- Consulta en sala y Consultas de revistas.

- Préstamo de libros colección general y Préstamo de libros colección reserva.

- Referencia electrónica (Internet y/o bases de datos).

- Servicios referencia (orientación en búsqueda de información).

De los resultados obtenidos en el análisis situacional se determina que el $54 \%$ de los servicios descritos anteriormente si se cumplen y el $46 \%$ no se cumplen; mientras que, luego de haber realizado la implementación del software ABCD y aplicado la encuesta a un grupo objetivo de 15 estudiantes y 5 profesores de las carreras, se obtiene como resultado que el $84 \%$ si se cumple y $16 \%$ no se cumple. Es muy notable el incremento en el uso de los servicios de la biblioteca; el software multiplicó las consultas a tesis, monografías, trabajos de investigación y libros de la biblioteca, además el sistema lleva un registro de la gestión diaria de la biblioteca en una misma base de datos con relación histórica de la progresión de los catálogos, es decir, de todas las altas, bajas, actualizaciones y consultas que se hagan desde el catálogo general de la biblioteca.

También, el software ABCD lleva un registro de los préstamos a los usuarios, este servicio implica un ciclo de préstamo, que es el préstamo propiamente dicho y la devolución. Los préstamos acarrean un registro de los libros, del usuario prestatario y de las políticas de préstamo. Para lo cual se crearon tablas para el alojamiento de los registros catalográficos, usuarios, perfiles de préstamo para definir las políticas de préstamo. Esto permitió tener un control completo de los catálogos bibliográficos de la biblioteca, así como de los usuarios que hacen uso de la documentación.

Además, la administración de los usuarios de la biblioteca, adquisiciones, políticas de préstamo que responden ante un determinado Marco normativo, Reglamento o Estatuto de gestión, Edición de estadísticas, Edición de la planificación y la difusión de comunicados a un público objetivo de la biblioteca.

\section{CONCLUSIONES}

En conclusión el software de Automatización de Bibliotecas y Centros de Documentación (ABCD) es ajustable a cualquier biblioteca ya que sus bases de datos tienen estandarizado los campos a ser utilizados en centros de documentación y su interfaz es amigable con el usuario, además es una herramienta bibliográfica flexible y versátil que facilita la gestión de los procesos de una biblioteca.

Con la implementación de los módulos de catalogación, adquisiciones, préstamos, devoluciones, consultas, estadísticas, reportes y administración de usuarios en la biblioteca 
de la Unidad Académica de Ingeniería de Sistemas, Eléctrica y Electrónica de la Universidad Católica de Cuenca, se centralizó la información en una misma base de datos conjuntamente con las tesis, monografías y trabajos de investigación. Esto incrementó el acceso de los usuarios al contenido bibliográfico, a través de consultas online, préstamos, referencias electrónicas y búsquedas mediante el internet.

Todas las bibliotecas deberían hacer uso de este software de código abierto para la automatización de sus procesos documentales y gestión de los mismos.

\section{AgRAdECIMIENTOS}

De manera muy especial expresamos un agradecimiento a la Universidad Católica de Cuenca, por brindarnos su apoyo para culminar esta investigación; también al equipo de investigadores conformado por los estudiantes: Joffre Alcisar Fajardo Rivera, Andrés Alfredo Gómez Urgiléz, Alex Javier Granda Pilacuán, Henry Jhonatan Tenesaca Mendía, Iván Mauricio Nieto Muñoz, a los docentes, estudiantes, personal administrativo y autoridades mil gracias por la oportunidad brindada, y por haber compartido sus conocimientos y experiencias.

\section{REFERENCIAS}

[1] P. Gethin, "Automatización de bibliotecas," El profesional de la información, vol. 10, pp. 26-31, Noviembre 2001.

[2] R. Osuna Alarcón and E. De la Cruz Gómez, "Los sistemas de gestión de contenidos en información y documentación/the contents management systems in information and documentation," Revista General de Información y Documentación, vol. 20, p. 67, 2010.

[3] B. S. Sánchez Vignau, "La universalización de la educaciòn superior en cuba: una oportunidad para reflexionar sobre los modelos de gestión en las bibliotecas universitarias," Acimed, vol. 12, no. 2, pp. 1-1, 2004.

[4] Biblioteconomía, "Sistemas integrados de automatización de bibliotecas. situación actual y tendencias de futuro.," Recuperado de: http://www.bibliopos.es/Biblion-A2-Biblioteconomial 23Sistemas-Integrados-Automatizacion-Bibliotecas. $p d f, 2013$.

[5] M. Breeding, "Tendencias actuales y futuras en tecnologías de la información para unidades de información," El profesional de la información, vol. 21, no. 9, pp. 9-15, 2012.

[6] R. L. A. D. M. S. K. Iglesias Cañedo, Carlos Manuel; Zamora Fonseca and K. Chaviano Nuñez, "La biblioteca virtual de la universidad de cienfuegos," Contribuciones a las Ciencias Sociales, no. 2010-03, 2010.

[7] J. A. Merlo Vega, "Fundamentos de gestión de bibliotecas universitarias," 1998.
[8] O. Arriola Navarrete and E. Montes de Oca Aguilar, "Sistemas integrales de automatización de bibliotecas: una descripción suscinta," Bibliotecas y Archivos, vol. 1, pp. 47-76, Noviembre 2014.

[9] O. Arriola Navarrete and G. Tecuatl Quechol, "Bibliotecas universitarias y automatización: un panorama de la ciudad de méxico," Revista Interamericana de Bibliotecología, vol. 34, pp. 129-146, Agosto 2011.

[10] P. Moscoso, "La nueva misión de las bibliotecas universitarias ante el espacio europeo de enseñanza superior," 2003.

[11] C. de Educación Superior, "Ley orgánica de educación superior," 2010.

[12] E. de Smet and E. Spinak, "El abc del abcd: Manual del modulo central," 2009.

[13] R. M. Merino, "Hacia la evaluación y acreditación de bibliotecas universitarias," 2013.

[14] Bireme, "Abcd," 2016.

[15] G. Fernández and N. Lenzo, "Software abcd (automatización de bibliotecas y centros de documentación): nuevos desafíos para las bibliotecas argentinas," 2010.

[16] E. De la Cruz Medina, D. G. Valdés, and E. C. Cabrera, "Implementación del sistema abcd en el crai de la universidad de cienfuegos," Biblios: Journal of Librarianship and Information Science, no. 58, pp. 6265, 2015.

Recibido: 09 de enero de 2017

Aceptado: 10 de marzo de 2017

Milton Alfredo Campoverde Molina: Ingeniero de Sistemas, Magíster en Docencia Universitaria, Magíster en Evaluación y Auditoria de Sistemas Tecnológicos. Profesor Titular e investigador de la Unidad Académica de Tecnologías de la Información y la Comunicación de la Universidad Católica de Cuenca - Ecuador.

Jenny Karina Vizñay Durán: Ingeniera de Sistemas, Especialista en Docencia Universitaria, Magíster en Evaluación y Auditoria de Sistemas Tecnológicos. Profesora Titular e investigadora de la Unidad Académica de Tecnologías de la Información y la Comunicación de la Universidad Católica de Cuenca - Ecuador. Correo eléctrónico: jviznay@ucacue.edu.ec

Silvia Eulalia Vintimilla Jara: Ingeniera de Sistemas, Magíster en Educación Mención Educación Superior MSC, Magíster en Ciencias de la Computación Mención Aplicaciones Distribuidas. Profesora Titular e investigadora de la Unidad Académica de Tecnologías de la Información y la Comunicación de la Universidad Católica de Cuenca Ecuador. Correo eléctrónico: svintimillaj@ucacue.edu.ec 